\title{
Ergonomic Review of the Processes of High-alert Medications for Hospital Inpatients
}

\author{
Sheau-Farn Max Liang(maxliang@ntut.edu.tw) \\ Department of Industrial Engineering and Management, National Taipei University of Technology, Taipei, Taiwan
}

\begin{abstract}
Medication error is the most frequent category of errors among others, according to the 2015 annual report of the Taiwan Patient-safety Reporting system (TPR). This type of errors is especially prevalent among hospital inpatients. For those inpatients treated with high-alert medications, any error in their treatments may cause serious adverse outcomes for them. The aim of this study is to review the process of high-alert medications for hospital inpatients with an emphasis on ergonomic perspective. First, a committee was formed consisting of physicians, pharmacists, nurses, and ergonomists. Then, the committee visited four medical institutes to observe their procedures of prescribing, dispensing, and administrating high-alert medications to inpatients. Next, physicians, pharmacists, and nurses in the institutes were interviewed for understanding their daily practices and related issues or problems. Finally, data collected from the observations and interviews were further discussed in the committee to identify room for further improvement in reducing errors and enhancing usability. The observations and interviews focused on the three major stages: physician order entry, pharmacy dispensing, and medication administration. In the stage of physician order entry, Computerized Physician Order Entry (CPOE) systems were used in all the institutes. However, all the systems were different in terms of their functionalities, user interfaces, and databases. Issues about information display, such as the size of order entry window, use of colors and blinks, alert display, and information visualization, were pointed out for further improvement. In the stage of pharmacy dispensing, the major issues were workplace layout and physical environment. Moreover, information could be more visualized. Finally, in the stage of medication administration, multitasking was the common practice and may lead to work overload. Therefore, simplifying procedures or providing aids may improve the work quality of nurses. Furthermore, labels on the medications and the display on the administration devices could be redesigned. Many ergonomic issues were found in the process of high-alert medications for hospital inpatients. These issues could increase mental workload and/or reduce situation awareness of these healthcare practitioners, and consequently might cause errors. In this preliminary study, we identified the problems in the medication process and provided possible solutions. Further empirical research on these identified ergonomic issues in the process of high-alert medications for hospital inpatients should be worthwhile.
\end{abstract}

Keywords: Medication error, Physician order entry, Pharmacy dispensing, Medication administration

\section{Introduction}

Medication error is the most frequent category of errors among others, according to the 2015 annual report of the Taiwan Patient-safety Reporting (TPR) system (Taiwan Joint Commission on Hospital Accreditation, 2015). TPR system was established in 2003 to encourage healthcare organizations and workers to report any clinical accidents and incidents voluntarily and anonymously.

For possible causes of medication errors, the majority of reporters thought the errors were related to practitioner factors, such as personal neglect and lack of training. Only a small portion of the reporters contributed the errors to the factors of work processes, equipment, communication, patients, or environment. These results were not surprising since an error usually involves people. However, this categorization cannot reflect the true causes of human errors because errors are caused by the interactions among human and other elements in the system.

In addition, most reporters suggested that personnel training as the primary solution to the medication errors. This is against the general human centered principle: Considering how systems fit people first, then vice versa. In brief, it appears to be a gap about the understanding of medication errors and associated solutions between 
healthcare and ergonomics specialties.

The aim of this study is to close the gap by conducting a preliminary review on current practices of medication. The annual report showed that medication errors are especially prevalent among hospital inpatients. Furthermore, for those inpatients treated with high-alert medications, any error in their treatments may cause serious adverse outcomes for them. Therefore, the medication process of high-alert medications for hospital inpatients was targeted and examined from an ergonomic perspective.

\section{Method}

A review committee was formed consisting of two physicians, one pharmacist, one nurse, and two ergonomists. Four medical institutes, two medical centers and two regional hospitals, were visited.

A whole day was spent during each visit. In the morning of each visit, the committee received a briefing on current practice from the institute before observing the procedures of prescribing, dispensing, and administrating high-alert medications to inpatients treated with chemotherapies. In the afternoon, the committee interviewed physicians, pharmacists, and nurses in the institute about their daily practices and related issues or problems.

Data collected from the observations and interviews were further discussed in the committee to identify room for improvement in reducing errors and enhancing usability. A debriefing session was provided to the institute at the end of the visit to confirm revealed issues and receive feedback from the institute. A final review report was delivered later to the four medical institutes for their reference.

\section{Results}

The observations and interviews focused on the three major stages of the high-alert medication process: physician order entry, pharmacy dispensing, and medication administration. Ergonomic issues found in each stage are discussed in the following sections.

\subsection{Physician order entry}

In the stage of physician order entry, only qualified physicians can prescribe high-alert medications at specific workstations with Computerized Physician Order Entry (CPOE) systems. CPOE systems were used in all the visited medical institutes though obvious deviations were found among these CPOE systems in terms of their functionalities, user interfaces, and databases. These systems were either PC-based or web-based applications. However, the typical WIMP (Windows, Icons, Menus, and Pointer) user interface was used in these systems. While a keyboard and a mouse were the common input devices, an LCD computer monitor was the information display device.

Basic functions were provided in all the systems. For example, preplanned medication regimens were available in CPOE systems so that physicians can retrieve a suitable regimen or modify an existed regimen instead of create a new one. Automatic calculations about medication doses were provided based on patient's body surface area derived from patient's height and weight so that physicians did not need to do the calculations manually. An alert function can issue a warning for possible drug interactions, abnormal doses, or any other potential risks concerned with the patient.

With computerized order entry, there are no more problems about physician's handwriting identification. Furthermore, these provided functions in CPOE systems could avoid and reduce significant medication errors. However, introducing a new system always brings new issues (Koppel et al., 2005). Four concerns about information display were pointed out in this review. They were (1) size of order entry window, (2) use of colors and blinks, (3) alert display, and (4) visualization.

Physicians opened a window on the screen to enter their orders. The format of the order entry window was like a table. Names of drugs were listed in rows and their associated information, such as medication doses and routes, was listed in following columns. However, the size of the window was not large enough. That is, physicians needed to use vertical or horizontal scrollbars to see the information in rows or columns that was out of the window. This made information access more difficult as well as needed more effort to keep the whole order in mind. To solve this problem, it was suggested to redesign the software or to use larger or multiple computer monitors so that a whole order can be display within a window. This problem has also been identified in previous research (Koppel et al., 2005).

We observed that it was common to use different colors to present different types of information in CPOE systems. However, it appeared that no specific design rules or 
guidelines about the use of colors in the systems. It was suggested that colors should be used with caution. Too many colors may make a display cluttered and complex for physicians to seek the information and understand the meanings of the colors. Color contrasts should also be considered in the design. Low color contrasts may not be able to distinguish presented information. Moreover, background is preferred in monochrome so that the important information in colors can be highlighted from the background. Finally, to avoid possible visual distraction, it was not suggested to use blinks, or at least they should only be used for essential information that needs immediate responses

During the process of physician order entry, we observed that alert dialog boxes were frequently popped up on the screen, but most of them were bypassed immediately by physicians. It was suggested that medical institutes should review their alert systems since too frequent pop-up dialog boxes might cause visual distraction and fatigue. Even worse, physicians may not pay enough attention to the alert dialog boxes and miss important information. In addition, the contents and formats of these alerts should be designed based on ergonomics principles (Russ et al., 2014).

The final issue of information display was about visualization. Current user interfaces of CPOE systems were text-based. Since double-check the physician order is a standard procedure, it was suggested to apply the design concept of configural display (Bennett and Flach, 1992) so that the visual double-check can be more efficient with graphical information displays.

\subsection{Pharmacy dispensing}

Pharmacy dispensing starts from the reception of physician order and includes medication preparation, packaging, and labeling. High-alert medications were prepared in an isolation room within the pharmacy. While some drugs might be prepackaged and ready to administer, others needed to be prepared in bags, bottles, or vials for matching patient-specific doses. Patient-specific medications were usually diluted with solutions, packaged, and labeled. Since the drug containers had fixed volumes, the drug doses in the physician order should consider the final volumes of liquid medications and allow pharmacists to prepare those medications under the limits of these container volumes.

Another important ergonomic issue in this stage was the workplace layout and physical environment of the pharmacies. Unlike the stage of physician order entry that most of jobs were done with computers, medications were passed around pharmacies and eventually delivered to nursing stations in sealed boxes.

According to physician orders, high-alert drugs were retrieved from lockers and sent to the isolated preparation room with a delivery cart. Once the medications were ready, the delivery cart was used again to bring back the medications. These medications were then put into a box and sealed with a barcode label.

Since there were many desks, computers, medical cabinets in pharmacies, workplace re-layout was suggested to improve the efficiency of traffic flows and avoid bad working postures. Furthermore, environmental noises, such as telephone rings, doorbells due to the access controls of pharmacies and preparation rooms, and noises from opening and closing drawers of medical cabinets, might distract pharmacists' attention. Further review of these noise sources was suggested.

Finally, since pharmacists needed to monitor patients' physiological conditions regularly, the configural display can also be useful to visualize the trends of patients' conditions.

\subsection{Medication administration}

For administering high-alert medications, such as drugs for chemotherapy, nurses needed to wear Personal Protective Equipment (PPE) first. Typical PPE included double gloves and protective gowns. Two nurses delivered drugs with a nursing trolley to a patient so that they can double-check the medications and administration procedures. The patient was identified by both asking his/her name and by scanning the barcode on his/her wristband.

A bedside smart infusion pump was used for delivering liquid medications to the patient's body in a controlled rate and duration. According to the information on the medication labels, nurses set up the rate and duration through the control panel on the pump. Therefore, it is important to provide correct and salient information on the label to facilitate the process of medication administration (Gerhart et al., 2015).

Since this is the last stage to intercept any medication error, many check procedures have been added into this stage. This may increase nurses' workloads and cause work fatigues (Roth et al., 2016). Furthermore, current practice was that each nurse took care of four to six patients. This is a typical multitasking or timesharing jobs. It is not possible to 
memorize the treatment status of each nursed patient. Currently many nurses took notes about patients' statuses in their own paper-and-pencil format. Therefore, a reminder system might assist nurses to handle all the treatments of their patients.

It was suggested that a handheld device, such as a tablet computer or mobile phone, might be used as the platform of the reminder system. An example is a reminder system built on a tablet computer to allow physical therapists to keep track of their patients' progresses of treatments (Liang et al., 2014). Information about each patient's location, time remained during a specific treatment were shown on the tablet computers. To build the system, it was suggested to gather and analyze nurses' needs in the medication administration through a user requirement analysis.

\section{Discussion}

Many ergonomic issues were found in the process of high-alert medications for hospital inpatients. These issues could increase mental workload and/or reduce situation awareness of these healthcare practitioners, and consequently might cause errors. In this preliminary study, we identified the problems in the medication process and suggested possible solutions.

In the stage of physician order entry, the main ergonomic issue was the interaction between physicians and CPOE systems. Suggestions were about user interface designs for a whole-page order entry window, alert dialog boxes, colors and blinks, and transforming texts into figures.

In the stage of pharmacy dispensing, workplace and environment were the major ergonomic issue. Suggestions focused on modifying workplace layout to make traffic flows more efficient, and reducing the distraction from environmental noises.

Finally, in the stage of medication administration, the main ergonomic issue was the workload derived from the nature of multitasking jobs. Suggestions emphasized on providing aids, such as reminders, to facilitate nurses to monitor the statuses of their patients.

Further empirical research on these identified ergonomic issues in the process of high-alert medications for hospital inpatients should be worthwhile.

\section{Acknowledgements}

This work was supported by Taiwan Joint Commission on Hospital Accreditation (TJCHA).

\section{References}

Bennett, K.B. and Flach, J.M., Graphical displays: Implications for divided attention, focused attention, and problem solving. Human Factors, 34(5), 513-533, 1992.

Gerhart, J.M., Spriggs, H., Hampton, T.W., Hoy, R.M.B., Strochlic, A.Y., Proulx, S., Goetchius, D.B., Applying human factors to develop an improved package design for $(\mathrm{Rx})$ medication drug labels in a pharmacy setting. Journal of Safety Research, 55, 177-184, 2015.

Koppel, R., Metlay, J.P., Cohen, A., Abaluck, B., Localio, A.R., Kimmel, S.E., Strom, B.L., Role of computerized physician order entry systems in facilitating medication errors. Journal of the American Medical Association, 293(10), 1197-1203, 2005.

Liang, S.F.M., Cheng, L.W., Tsai, P.F.J., Rau, C.L., "Designing user interfaces of a handheld device for physical therapists", In: Proceedings of the $4^{\text {th }}$ International Conference on Healthcare Systems Ergonomics and Patient Safety, (6 pages), Taipei, Taiwan, 2014.

Roth, C., Brewer, M., Wieck, K.L., Using a Delphi method to identify human factors contributing to nursing errors. Nursing Forum, doi:10.1111/nuf.12178, 2016.

Russ, A.L., Zillich, A.J., Melton, B.L., Russell, S.A., Chen, S., Spina, J.R., Weiner, M., Johnson, E.G., Daggy, J.K., McManus, M.S., Hawsey, J.M., Puleo, A.G., Doebbeling, B.N., Saleem, J.J., Applying human factors principles to alert design increases efficiency and reduces prescribing errors in a scenario-based simulation. Journal of the American Medical Informatics Association, 21, e287-e296, 2014.

Taiwan Joint Commission on Hospital Accreditation (TJCHA), Annual Report of Taiwan Patient Safety Reporting System (in Chinese), 2015, http://www.patientsafety.mohw.gov.tw/Content/Downloads/List01.as px?SiteID=1\&MmmID=621273303702500244 (retrieved March 19, 2017). 